\title{
Convergent-flow tracer tests in heterogeneous media: combined experimental-numerical analysis for determination of equivalent transport parameters
}

\author{
Daniel Fernàndez-Garcia ${ }^{\mathrm{a}, *}$, Xavier Sánchez-Vila ${ }^{\mathrm{b}}$, \\ Tissa H. Illangasekare ${ }^{\mathrm{a}}$ \\ ${ }^{a}$ Environmental Science and Engineering Division, Colorado School of Mines, 1500 Illinois, \\ Golden, CO 80401, USA \\ ${ }^{\mathrm{b}}$ Department of Geotechnical Engineering and Geosciences, Technical University of Catalonia, \\ Gran Capita S/N, 08034 Barcelona, Spain
}

Received 16 February 2001; received in revised form 25 October 2001; accepted 16 November 2001

\begin{abstract}
In modeling transport within naturally heterogeneous aquifers, it is usually assumed that the transport equations valid at local scales can also be applied at larger scales. At larger scales, the heterogeneous domain is represented by an equivalent homogeneous medium. Convergent-flow tracer tests constitute one of the most frequently used field tests to estimate effective input parameters of equivalent homogeneous aquifers. Traditionally, statistical approaches applied to groundwater flow and solute transport have provided tools to estimate these equivalent parameters. These approaches are based on a number of simplifications including the assumption that the point transmissivity values follow a multilog-normal random function. Several investigators have found that this assumption may not be valid in many field cases. In order to study the applicability of the equivalent homogeneous formulation in a nontraditional stochastic field, a number of experimental and numerical studies were conducted. The results are used to determine the apparent values of porosity and dispersivity that would be obtained if convergent-flow tracer tests were conducted in a deterministically generated heterogeneous transmissivity field displaying anisotropy in the correlation structure. It is shown that in this particular heterogeneous media, apparent porosity strongly depends on connectivity rather than on transmissivity. This dependence on connectivity
\end{abstract}

\footnotetext{
* Corresponding author. Tel.: +1-303-384-2237; fax: +1-303-273-3629.

E-mail addresses: dfgarcia@mines.edu (D. Fernàndez-Garcia), xavier.sanchez-vila@upc.es (X. Sánchez-Vila), tillanga@mines.edu (T.H. Illangasekare).
} 
questions the theoretical results obtained in continuum equivalent fields to estimate effective porosity. (C) 2002 Elsevier Science B.V. All rights reserved.

Keywords: Solute transport; Porous media; Heterogeneity; Spatial variability; Porosity; Dispersion

\section{Introduction}

Numerical models designed to simulate the flow of water and solute migration through aquifers require the discretization of the solution domain into blocks or elements defined by a computational grid. As a result, the computational-scale used in these models usually becomes much larger than the representative elemental volume (REV) scale where the classical governing groundwater flow and solute transport equations are strictly applicable. To overcome this difficulty, it is traditionally considered possible to describe the heterogeneous porous formation defined at the grid-scale by means of an equivalent homogeneous medium. The properties attributed to this virtual medium are known as equivalent or block parameters, and they can be seen as spatial averages of the local properties over the computational grid-scale (Dagan, 1986; Desbarats, 1992). Following this approach, it is

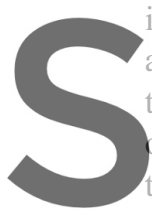
implicitly assumed t. approximation to the 1 to groundwater flow

of the average solution these so-called effective
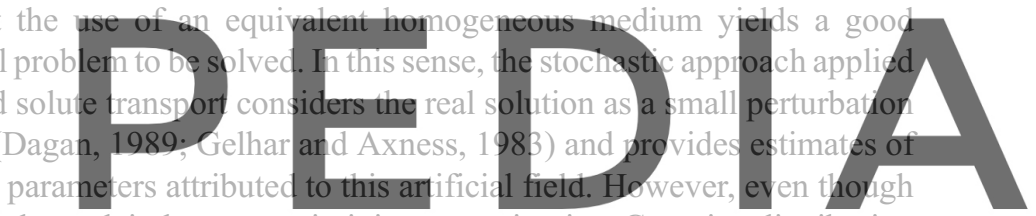

field data can only properly explain log-transmissivity as a univariate Gaussian distribution

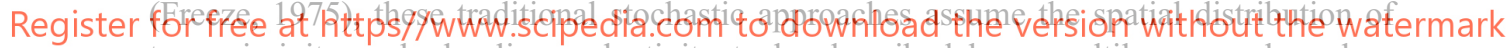
transmissivity or hydraulic conductivity to be described by a multilog-normal random function. Recent investigations have studied the magnitude of this extrapolation and found that real field sites do not display many of the characteristics of multi-Gaussian distributions (Gómez-Hernández and Wen, 1996; Sánchez-Vila et al., 1996).

The main objective of this paper is to further investigate the applicability of equivalent homogeneous fields in a more realistic context as well as to evaluate those transport parameter estimates resulting from the interpretation of tracer tests based upon equivalent homogeneous fields. The analysis is limited to the aquifer parameters typically obtained from convergent radial flow tests, which are porosity and longitudinal dispersivity. The spatial variability of these two parameters is investigated in a heterogeneous, nonstochastically generated domain that displays anisotropy in the correlation structure of the transmissivity field (T-field). In convergent radial flow tests, water is pumped from a single well until steady-state flow is achieved. A tracer is then introduced into a well located at some distance from the pumping well and the breakthrough tracer concentrations at the pumping well are recorded as a function of time. Interpretation of the tracer breakthrough curve (BTC) under the assumption of homogeneity and radial symmetry yields the required aquifer parameters. However, since natural aquifers are heterogeneous, these parameter values obtained from ideal isotropic models vary as a function of the injection location. These parameters can only be seen as fitting or apparent parameters, and they should be treated as 
spatial variables. From this standpoint, the question arises on what exact parameters are estimated from convergent-flow tracer tests BTCs that are interpreted assuming aquifer homogeneity.

A combined experimental and numerical investigation was conducted to address this question. In the experimental component of the study, several convergent-flow tracer tests were conducted in a heterogeneously packed, two-dimensional horizontal soil tank. The tank was packed with four different test sands to represent a deterministically generated heterogeneous field. As experimental simulations are very tedious and time-consuming, the data from a limited number of experiments were utilized to validate a numerical model that was then used to perform additional computational analyses. Simulated tracer tests were used to obtain the apparent values of porosity and dispersivity using a simple methodology valid for homogeneous isotropic media. All the tracer test simulations were run in a single realization of the heterogeneous domain, as we are only interested in actual equivalent parameters and not in statistical mean values.

\section{Theoretical background}

\subsection{Working assumptions and limitations}
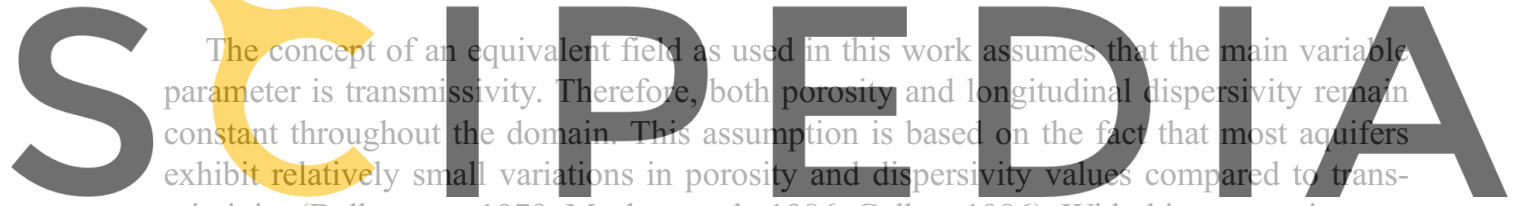

missivity (Delhomme, 1979; Mackay et al., 1986; Gelhar, 1986). With this assumption, we

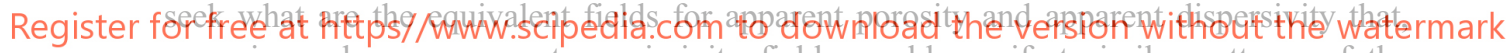
assuming a homogeneous transmissivity field, would manifest similar patterns of the breakthrough curves in convergent-flow tracer tests.

\subsection{Spatial variability of apparent parameters in heterogeneous T-fields perfectly repre- sented by an equivalent medium}

In order to investigate the applicability of equivalent fields to the interpretation of convergent-flow tracer test BTCs, it is necessary to understand first the spatial variability that these apparent parameters would ideally display in heterogeneous T-fields that are perfectly represented by an equivalent medium. Within this context, Sánchez-Vila and Carrera (1997) showed that the porosity obtained by interpreting convergent tracer BTCs in heterogeneous T-fields with anisotropic spatial correlations is not homogeneous but it has directional properties; porosity delineates an ellipse perpendicular to the constantcorrelation ellipse of the T-field. Apparent porosity was found to be better represented by a second order symmetric tensor with main attributes: (1) real effective porosity falls between the two values of the principal components; and (2) the geometric mean of the principal components yields the true porosity (assumed constant). Moreover, Sánchez-Vila and Carrera (1997) showed that regions of equal travel times also delineate ellipses, but they are parallel to the constant-correlation ellipse of the T-field. 
Spatial variability of apparent dispersivity is not that well understood for convergent radial flow in anisotropically correlated T-fields. However, since 'macrodispersivity' is found to be a second rank symmetric tensor in uniform flow (Gelhar and Axness, 1983), it is assumed that apparent dispersivity also follows this type of elliptical directional behavior.

\subsection{Interpretation of breakthrough curves}

A number of analytical and numerical solutions to the transport equation for convergent radial dispersion under steady groundwater flow have been previously described in literature. Gelhar and Collins (1971) used two-coordinate integral transformations to reduce the formulation to the diffusion equation and obtain an approximate analytical solution useful for transport conditions with Peclet numbers greater than 15. Moench (1989) used the Laplace transform to obtain solutions using numerical inversion. Other authors solved the problem numerically and expressed the results as dimensionless breakthrough curves (Sauty, 1980; Carrera and Walters, 1985). However, these solutions were too cumbersome to be implemented into a transport code to conduct large number of simulations with changes in injection points. For this reason, the method of moments modified for radial flow was used in this analysis. This method of analysis is commonly

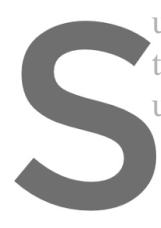
used in the interpreta this method, apparent

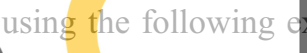
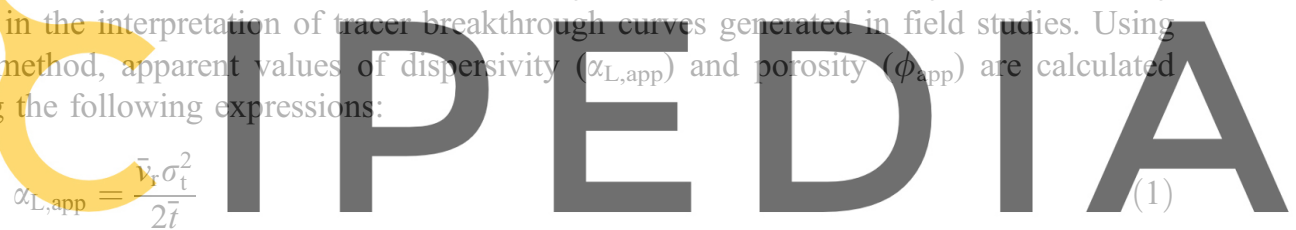

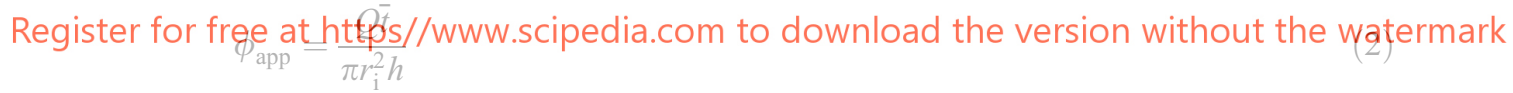

where $\bar{v}_{\mathrm{r}}$ is the average pore velocity between the point of injection and the well, $\bar{t}$ is the first moment or arrival time (mean arrival time), $\sigma_{\mathrm{t}}^{2}$ is the second temporal moment, $Q$ is the pumping rate, $r_{\mathrm{i}}$ is the distance between the injection and the pumping wells, and $h$ is the aquifer thickness. These parameters are found from the breakthrough curve of the tracer concentration $(C(t))$ using:

$$
\begin{aligned}
& \bar{t}=\frac{\int t C(t) \mathrm{d} t}{\int C(t) \mathrm{d} t} \\
& \sigma_{\mathrm{t}}^{2}=\frac{\int(t-\bar{t})^{2} C(t) \mathrm{d} t}{\int C(t) \mathrm{d} t}=\frac{\int t^{2} C(t) \mathrm{d} t}{\int C(t) \mathrm{d} t}-[\bar{t}]^{2} \\
& \bar{v}_{\mathrm{r}}=\frac{r_{\mathrm{i}}}{t}
\end{aligned}
$$


Convergent-flow tracer tests have the advantage that all flow lines converge to the pumping well and that the total mass of injected tracer is recovered in the extraction well (mass conservation). Mass recovery can be measured by:

$$
\mathrm{MR}=\int Q C(t) \mathrm{d} t / M_{0}
$$

where $M_{0}$ is the mass of tracer injected.

\subsection{Generation of the transmissivity field}

The heterogeneous T-field that was used in both experimental and numerical simulations of the convergent tracer tests was generated deterministically. As was discussed earlier, this avoided the need to build multi-Gaussian fields that are commonly used in numerical studies involving heterogeneous porous media (e.g. Gómez-Hernández and Gorelick, 1989; Tompson and Gelhar, 1990; Desbarats, 1992; Chao et al., 2000). In order to create the heterogeneous packing configuration in the laboratory, the transmissivity field was treated as a discrete field represented by four different types of well-characterized test sands. The test sands were distributed throughout a grid formed by $36 \times 16$ blocks $(6.096 \mathrm{~cm}$ square), each block containing a single sand type to represent a target conceptual model for variation of transmissivity in a variation: (1) the spatia the $y$-direction (anisotr not drastically differen this, rectangular lense
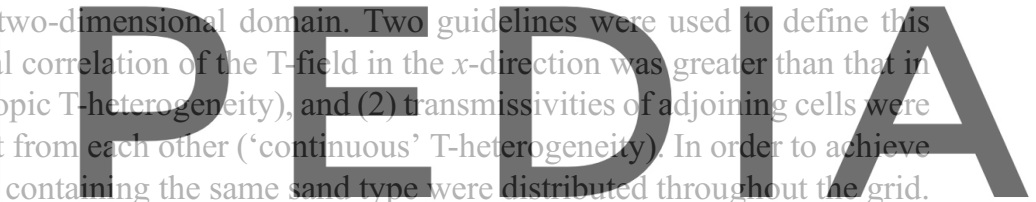

These lenses were designed with the $x$-dimension greater than the $y$-dimension, with a length

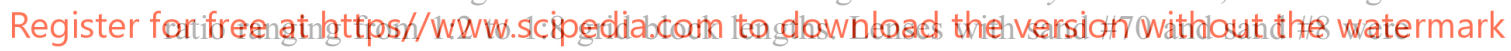
packed first, thus constraining them not to be in contact with each other (in compliance with guideline 2). Lenses with the other two sands $\# 16$ and $\# 30$ filled the remaining grid spaces in rectangular configurations. This process of sand placement led to the transmissivity field shown in Fig. 1. The test sands utilized in this analysis are dominated by silica minerals and

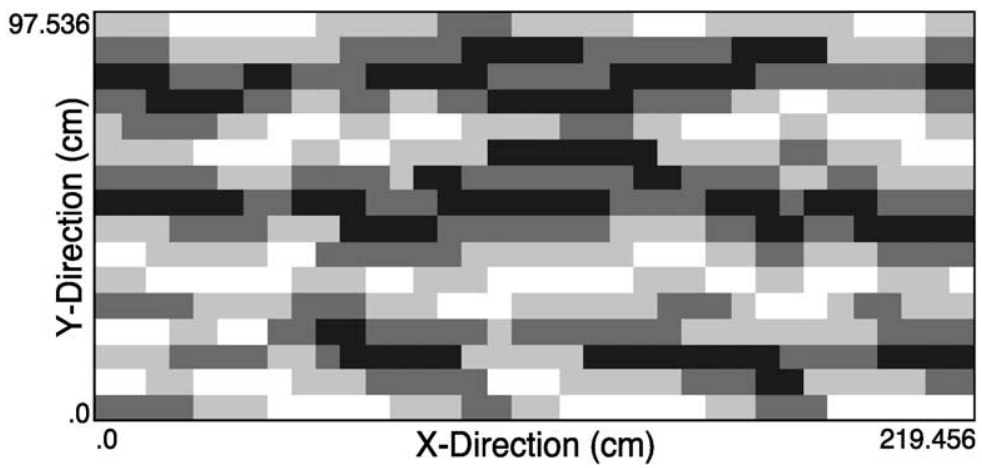

\section{SAND TYPE}

\# 8

$\square \# 16$

$\square$ \# 30

$\square$ \# 70

Fig. 1. Transmissivity field used in experimental and numerical simulations. 
Table 1

Properties of test sands

\begin{tabular}{lllllr}
\hline Sand no. & $D_{60} / D_{10}$ & $D_{10}(\mathrm{~mm})$ & $\phi$ & $\alpha_{\mathrm{L}}(\mathrm{cm})$ & $K(\mathrm{~m} /$ day $)$ \\
\hline 8 & 1.7 & 0.9 & $0.42-0.45$ & 0.12 & 1036.8 \\
16 & 2.0 & 0.7 & $0.42-0.45$ & 0.1 & 371.5 \\
30 & 1.6 & 0.38 & $0.42-0.45$ & 0.1 & 110.8 \\
70 & 1.9 & 0.15 & $0.42-0.45$ & 0.07 & 31.9 \\
\hline
\end{tabular}

$D_{60}, D_{10}$ : grain sizes from sieve analysis; $60 \%$ and $10 \%$ undersize by weight, respectively (Fernàndez-Garcia, 1999).

$\alpha_{\mathrm{L}}, K$ : longitudinal dispersivity and hydraulic conductivity from column experiments, respectively. $\phi$ : porosity obtained from both gravimetric measurements and column tracer experiments (Chao, 2000).

they have been well characterized in our past investigations (Illangasekare et al., 1995; Chao et al., 2000; Barth et al., 2001). The relevant properties of the test sands are given in Table 1. It should be noted that even though the mean grain sizes vary within a wide range, the porosities do not change drastically due to the uniformity of the test sands. The resulting transmissivity field of the packed tank has a discrete distribution in log-hydraulic conductivity space, with a mean of 5.2 ( $K$ in $\mathrm{m} /$ day $)$ and a variance of 1.47 .
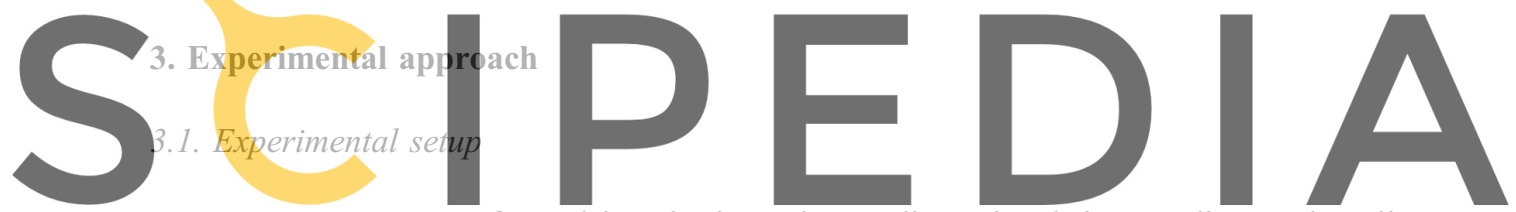

Tracer tests were performed in a horizontal, two-dimensional, intermediate-scale soil

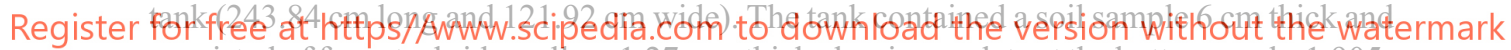
consisted of four steel side walls, a 1.27-cm-thick aluminum plate at the bottom, and a 1.905 $\mathrm{cm}$ transparent Plexiglas sheet at the top (Fig, 2). Constant head spill reservoirs connected to the upstream and downstream end of the tank were used to control the flow through the outer boundaries of the sand pack. The water entering the tank was distributed around the sand pack to create constant head boundary conditions needed for radial flow tests (Fig. 2). The transparent Plexiglas top allowed for the observation of dyed tracers and permitted the introduction of 48 pressure measurement ports as well as 29 injection and sampling ports. Each port was fitted with a three-way valve and a sampling needle that allowed for the extraction of water samples at mid-depth of the sand sample $(2.5 \mathrm{~cm}$ from the top wall). The pressure ports were connected to a transducer (Model P300D) through a scanning fluid switch. A computer automated solenoid controller was used to rotate the scanning value that connected the pressure transducer to each of the 48 pressure ports sequentially. The heterogeneous sand packing depicted in Fig. 1 was reproduced in the tank using a metal grid constructed with $0.15 \mathrm{~cm}$ tin sheets that form a rectangular mesh of $36 \times 16$ cells (to give square cells of side $6.096 \mathrm{~cm}$ ). Each grid cell was dry packed with the appropriate sand. The metal sheets were then extracted without disturbing the sand and the Plexiglas top was placed to confine the sand and seal the tank. The dry packed sand was then slowly flushed with carbon dioxide at $2.5 \mathrm{psi}$ for $24 \mathrm{~h}$ to evacuate the air. De-aired water was then introduced slowly through the supply tanks to displace the carbon dioxide. The water is expected to 
(a)

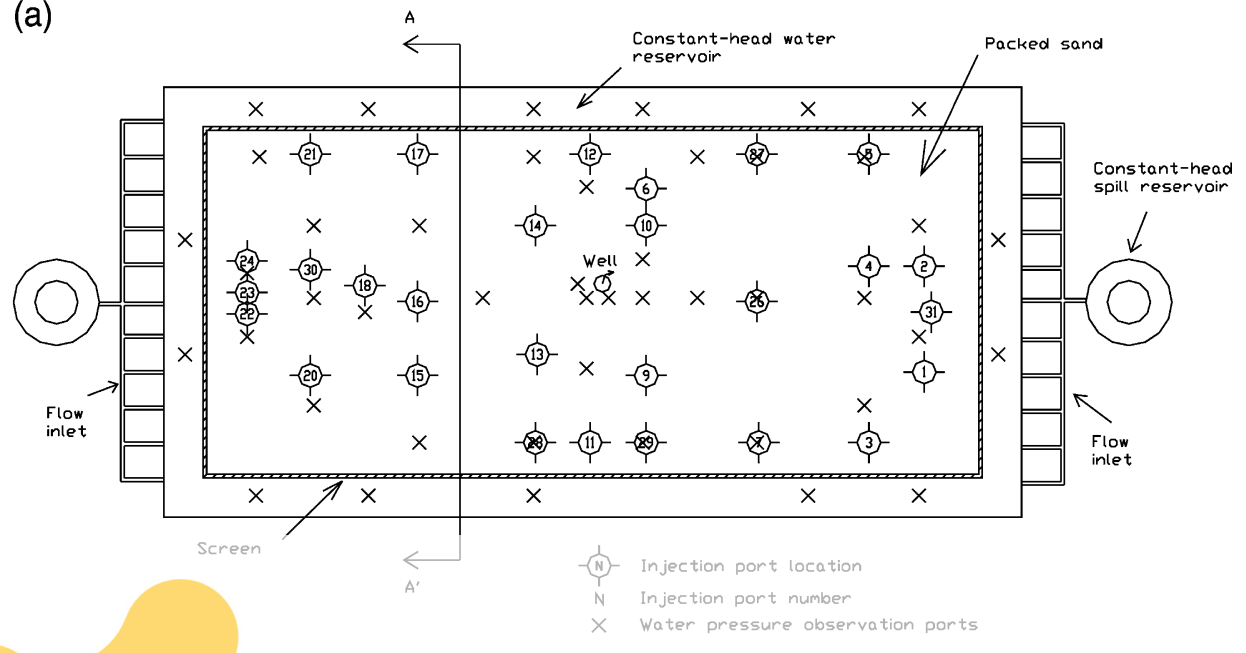

(b)
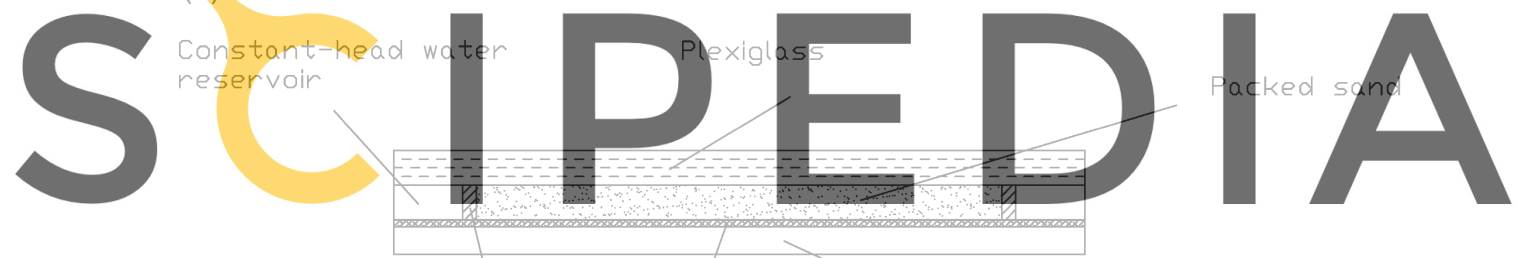

Register for free at https//wmw.scipedia.com to download the version without the watermark

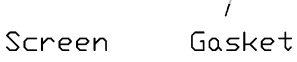

Fig. 2. Schematic of the sand tank. (a) Plan view with injection and observation ports lay-out. (b) $A-A^{\prime}$ cross section.

dissolve any entrapped gas to produce full saturation within the soil. Creating full saturation within the sample is critical as any entrapped air can significantly reduce the hydraulic conductivity of the sand.

\subsection{Experiments}

Prior to the initialization of the tracer tests, flow conditions were verified by monitoring water pressures at each observation port for $48 \mathrm{~h}$. A total of 28 convergent-flow tracer tests were performed. For each test, once steady-state flow was established, a slug of bromide tracer was injected over $15 \mathrm{~s}$ (injection locations are shown in Fig. 2a). During the test, water samples $(3 \mathrm{ml})$ were collected from an outlet sampling port located at the pumping well. Bromide concentrations in the collected aqueous samples were measured with a bromide 
probe (Orion model 9635). The boundary head and the pumping rate in the well were monitored over the course of the experiment to ensure that they remained constant. Table 2 provides the relevant conditions of the tracer tests and the transport parameters interpreted from the tracer breakthrough curves. As shown in this table, seven of the experimental BTCs exhibited bimodal behavior. This behavior was attributed to procedural errors in the way the tracer was injected. Hence, the data from these tests were not used in the analysis. The average mass recovery (MR) for all the experiments was $95 \%$, with a minimum value of $75 \%$ at the injection port $\# 15$. Those experiments with MR values less than $100 \%$ were attributed to a poor definition of the tail of the BTC due to both inaccuracies of the bromide probe at low concentrations and an unfinished recovery of the bromide tracer in cases where the tail was long. High recovery indicated that any significant mass of the tracer did not leave the boundaries. Peclet numbers associated to the tracer tests (defined as the ratio between radial distance and apparent dispersivity, see Table 2) ranged from 15 to 400.

After the experiments were completed, eight core samples (two for each test sand) were extracted at random locations. The in-situ porosities of the extracted samples were

\section{Table 2}

Summary of experiments

Injection $\quad C_{0}(\mathrm{mg} / \mathrm{l}) \quad V_{0}(\mathrm{ml}) \quad Q(\mathrm{ml} / \mathrm{min}) \quad \mathrm{MR}(\%) \quad \bar{t}(\mathrm{~min}) \quad \phi_{\text {app }} \quad \alpha_{\mathrm{L}, \mathrm{app}} \quad r_{\mathrm{i}} / \alpha_{\mathrm{L}, \mathrm{app}}$

port no.
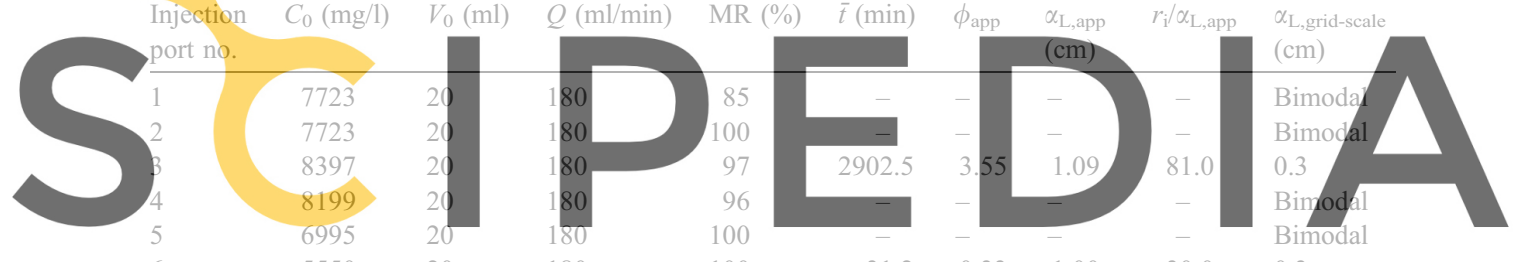

$5550 \quad 20 \quad 180 \quad 100$

Register for free ats łattps//20ww.scipedia.coms to

\begin{tabular}{rrrrrrrrrr}
9 & 5550 & 20 & 180 & 100 & 124.6 & 1.60 & 0.32 & 85.3 & 0.3 \\
10 & 240 & 15 & 75 & 86 & 37.4 & 0.34 & 0.38 & 55.2 & 0.3 \\
11 & 1074 & 20 & 180 & 86 & 418.7 & 1.97 & 0.24 & 187.9 & 0.3 \\
12 & 434 & 20 & 75 & 90.3 & 112.5 & 0.34 & 0.30 & 120.3 & 0.1 \\
13 & 5550 & 20 & 180 & 100 & 90.0 & 0.95 & 0.56 & 53.6 & 0.3 \\
14 & 5550 & 20 & 180 & 98.2 & - & - & - & - & Bimodal \\
15 & 221 & 15 & 75 & 75 & 299.4 & 0.38 & 0.14 & 399.2 & 0.1 \\
16 & 275 & 20 & 75 & 97 & 157.1 & 0.24 & 0.44 & 115.2 & 0.3 \\
17 & 5550 & 20 & 180 & 100 & 354.9 & 0.88 & 1.31 & 47.3 & 0.3 \\
18 & 5550 & 20 & 180 & 100 & 129.6 & 0.28 & 2.22 & 29.7 & 0.3 \\
20 & 8531 & 20 & 180 & 97 & 739.6 & 1.00 & 2.88 & 29.1 & 0.3 \\
21 & 9626 & 20 & 180 & 100 & 1119.9 & 1.38 & 3.52 & 25.0 & 1.1 \\
22 & 9691 & 20 & 180 & 100 & - & - & - & - & Bimodal \\
23 & 1053 & 20 & 180 & 100 & 301.3 & 0.29 & 6.76 & 14.7 & 1.3 \\
24 & 5458 & 20 & 180 & 92 & 309.4 & 0.30 & 3.91 & 25.5 & 0.3 \\
26 & 3423 & 20 & 180 & 98 & 75.3 & 0.34 & 1.37 & 33.3 & 0.3 \\
27 & 2634 & 20 & 180 & 99 & 119.9 & 0.34 & 0.72 & 80.5 & 0.3 \\
28 & 9626 & 20 & 180 & 100 & 299.4 & 1.22 & 1.60 & 30.3 & 0.3 \\
29 & 5569 & 20 & 180 & 94 & 337.9 & 1.47 & 0.99 & 47.3 & 0.5 \\
30 & 5894 & 20 & 180 & 90 & - & - & - & - & Bimodal \\
31 & 5550 & 20 & 180 & 100 & 272.6 & 0.29 & 4.84 & 19.7 & 0.9 \\
\hline
\end{tabular}

$V_{0}, C_{0}$ : volume and concentration of tracer injected, respectively. 


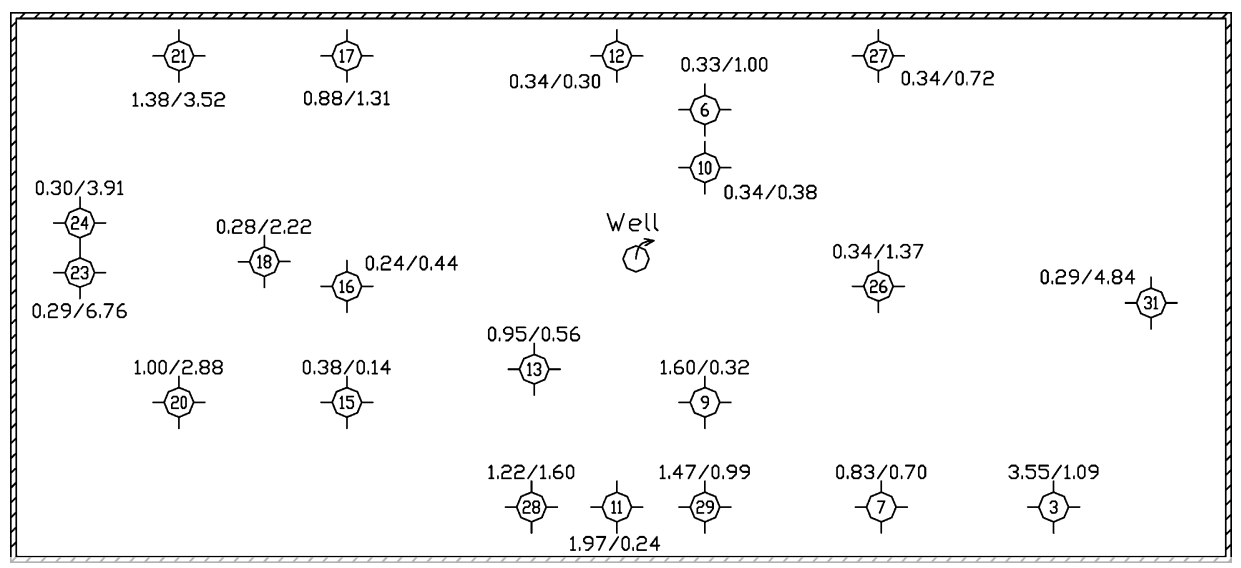

Fig. 3. Location map of porosity and dispersivity apparent values obtained from experimental BTCs $\left(\phi_{\text {app }}, \alpha_{\mathrm{L}, \text { app }}\right)$.

determined gravimetrically. The porosities were in the range 0.38 to 0.43 . The maximum porosity is within the range of the characterized values, but the minimum porosity is out of range (Table 1), indicating that the exact porosity was dependent on the packing method.
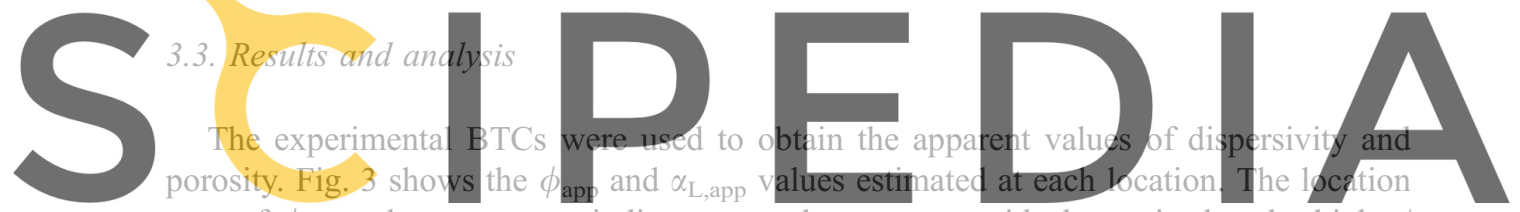

map of $\phi_{\text {app }}$ values seems to indicate a good agreement with theory in that the higher/

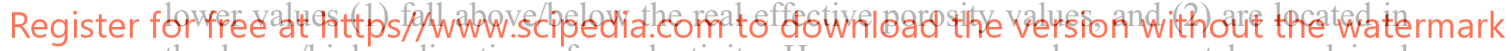

the lower/higher direction of conductivity. However, some values cannot be explained using the continuum equivalent theory. Ports \#3 and \#20 show values of apparent porosity that are much higher than values at adjoining locations, breaking down the otherwise smooth spatial continuity. This phenomenon is explained by realizing that these two data points are located in zones of low connectivity (the concept of connectivity is discussed in the computational investigations). The dispersivities show that even though $\alpha_{\mathrm{L} \text {,app }}$ values behave as expected (they increase with distance from the well and are always higher than the grid-scale values), they display higher values in the direction of higher transmissivity, where the flow paths are presumably less tortuous. This phenomenon was also observed during the numerical investigations carried out by Sánchez-Vila and Carrera (1997), who simulated radial flow tests in a multilog normally distributed stochastic random field.

\section{Computational approach}

\subsection{Selection of numerical models}

Disregarding small variations within particular sand blocks, the geometry of the heterogeneous T-field is fully known from the tank construction. Therefore, numerical 
models should be able to accurately reproduce the BTCs. A finite-element code, FAITH (Sánchez-Vila et al., 1993), was used to solve the flow equation. As the externally measured dispersivities of the individual test sands were very small, the relative contribution of numerical dispersion is expected to produce significant simulation errors if an Eulerian framework was selected to solve the transport equation numerically. Hence, a Lagrangian transport code (TRANSP) was selected to solve the advection-dispersion equation. The transport code TRANSP (Wen, 1995) solves the two-dimensional solute transport equation based on the Random Walk Particle Method (Kinzelbach, 1986; Tompson and Gelhar, 1990). This code not only accounts for the corrections to pore velocity proposed by Kinzelbach (1986) to ensure that the injected particles exactly reproduce the governing equation of transport but also incorporates a Constant Displacement (CD) algorithm (Wen, 1995).

The Random Walk Particle Method simulates solute transport by injecting a large number of particles into the system, each of which is representative of a portion of the solute mass. Moving particles simulates the advection mechanism, whereas the dispersion is represented using Brownian motion of the particles. In traditional random walk algorithms, the particles are moved by selecting the same incremental time for all particles at every time step (by means of, for example, the Courant number). However, in the CD algorithm used in this model, the movement of particles may also be achieved by specifying the number of

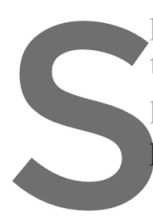
possible particle displaceme traveling very fast thro per cell). The CD appio particles are subjected to
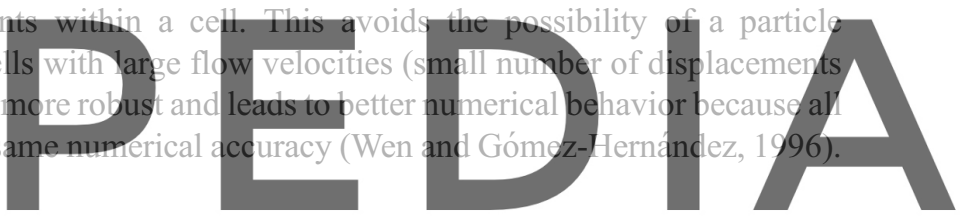

Simulation features

Register for free at https//www.scipedia.com to download the version without the watermark

The boundary conditions (constant head along the four sides of the domain and constant pumping rate) imposed during simulations correspond to the experimental investigations. The numerical grid used in the simulations consisted of $160 \times 360$ cells (squares of side $0.6096 \mathrm{~cm}$ ) and the transmissivity values were assigned using the sand packing distribution depicted in Fig. 1. A total of 476 convergent-flow tracer tests were simulated using the numerical model. Each test represented a different tracer injection location. Injection points were distributed throughout the domain by following a uniform rectangular grid of $34 \times 14$ (the injection points correspond to the geometric center of the sand blocks, and blocks at the boundaries were not considered). Following the $\mathrm{CD}$ algorithm, all particles were imposed to take 60 steps within each grid cell. Incorporation of a nonpunctual source was necessary to adequately reproduce the experimental data: initially, particles were distributed uniformly in space in a rectangular area centered at the injection port. This area was estimated by using a dye tracer and is assumed to account for the initial volume of tracer added into the system $\left(V_{0}\right)$ as well as for the increase in pressure during injection.

\subsection{Model calibration and validation}

The model was validated prior to its application using the laboratory data generated in the intermediate-scale tank. Head values simulated using the experimentally determined 
transmissivities matched well with the observed heads as shown in Fig. 4a. The transport model was calibrated by adjusting the porosity $\left(\phi_{\text {grid-scale }}\right)$ and the dispersivity coefficient $\left(\alpha_{\mathrm{L}, \text { grid-scale }}\right)$ of packed blocks. As discussed in Section 2.1, these two parameters were assumed to be constant within the entire domain. Since numerically simulated concentrations representing the experiments were found to be not sensitive to transverse dispersivity, no attempt was made to fit an anisotropic dispersivity. Transverse dispersivity at the grid-

(a)

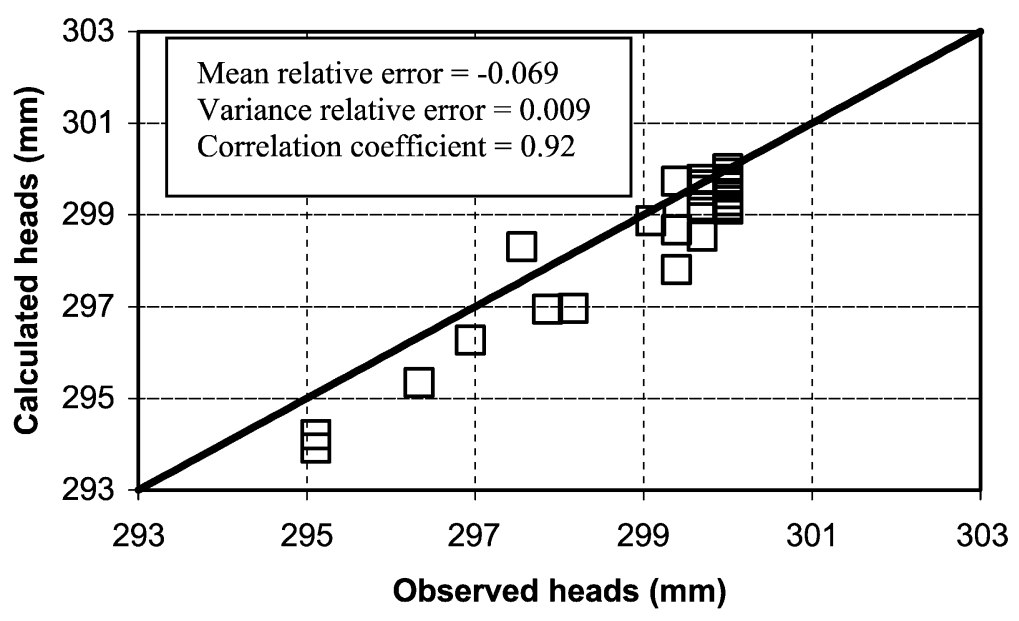

(b)

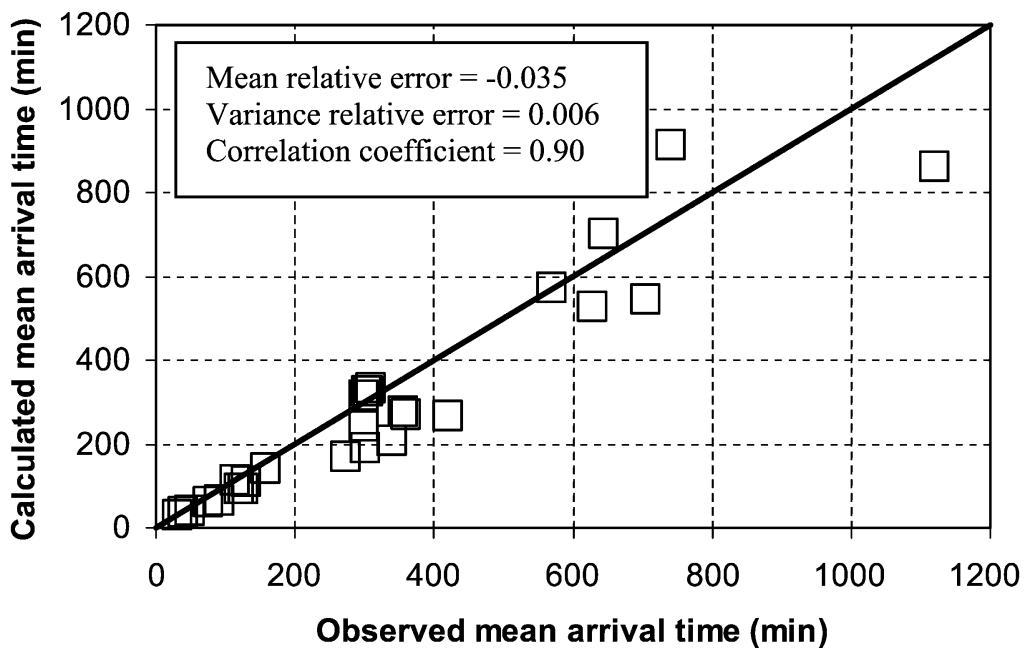

Fig. 4. Model calibration. (a) Comparison of calculated and observed water heads. (b) Comparison of calculated and observed mean arrival times. 
scale was assumed to be $0.014 \mathrm{~cm}$ in the computational investigations. Grid-scale porosity, $\phi_{\text {grid-scale, was determined by matching observed and calculated travel times whereas }}$ $\alpha_{\mathrm{L} \text {,grid-scale }}$ was obtained by reproducing the experimental BTCs. First, $\phi_{\text {grid-scale }}$ was adjusted until experimental travel times were reproduced (Fig. 4b). This process gave a porosity value of $\phi_{\text {grid-scale }}=0.44$, which is in agreement with test sand properties presented in Table 1 and is reasonably close to values obtained from core samples removed after disassembly of the tank. The calibration of $\alpha_{\mathrm{L} \text {,grid-scale }}$ was then conducted by curve matching the experimental BTCs with the numerical solutions. Fig. 5 shows a close match between the observations and calibrated model. The last column of Table 2 gives the calibrated $\alpha_{\mathrm{L} \text {,grid-scale values. It is }}$ crucial not to confuse the calibrated $\alpha_{\mathrm{L}, \text { grid-scale }}$ and $\phi_{\text {grid-scale }}$ with apparent values, $\phi_{\text {app }}$ and $\alpha_{\mathrm{L} \text {,app }}$. The grid-scale parameters are spatially constant properties that are obtained by curve matching the numerical solution that incorporates the perfectly known heterogeneous $\mathrm{T}$ field. On the contrary, the apparent parameters are spatially varying properties that are obtained assuming isotropic homogeneity (Section 2.3). For instance, the $\alpha_{\mathrm{L} \text {,grid-scale }}$ expresses the mixing mechanism at the REV scale whereas the $\alpha_{\mathrm{L}, \text { app }}$ is its upscaled value that includes the mixing effects caused by the spatial variability in transmissivity. Fifteen out of twenty-one BTCs were successfully reproduced with the same grid-scale dispersivity $\left(\alpha_{\mathrm{L}, \text { grid-scale }}=0.3 \mathrm{~cm}\right)$. This value was higher than the one obtained from column experiments (Table 1). This effect was partially attributed to the role of other mixing mechanisms such as the spatial variability of $\alpha_{\mathrm{L} \text {,grid-scale }}$ and $\phi_{\text {grid-scale, which were assumed constant during the }}$ numerical investigations. The other six experimental BTCs required small changes in $\alpha_{\mathrm{L}, \text { grid-scale }}$ to obtain a good match; injections \#21, \#23, \#29 and \#31 needed higher $\alpha_{\mathrm{L} \text {,grid-scale }}$ values whereas injections $\# 12$ and \#15 needed lower $\alpha_{\mathrm{L} \text {,grid-scale }}$ values; low values were very similar to the properties of the tests sands as presented in Table 1 and may correspond to solute pathways with insignificant variations of $\alpha_{L \text {,grid-scale }}$ and $\phi_{\text {grid-scale }}$. In reverse, high values may indicate pathways in which the effect of variations in $\alpha_{\mathrm{L} \text {,grid-scale }}$ and $\phi_{\text {grid-scale }}$ were effectively important. In overall, these results indicated that the

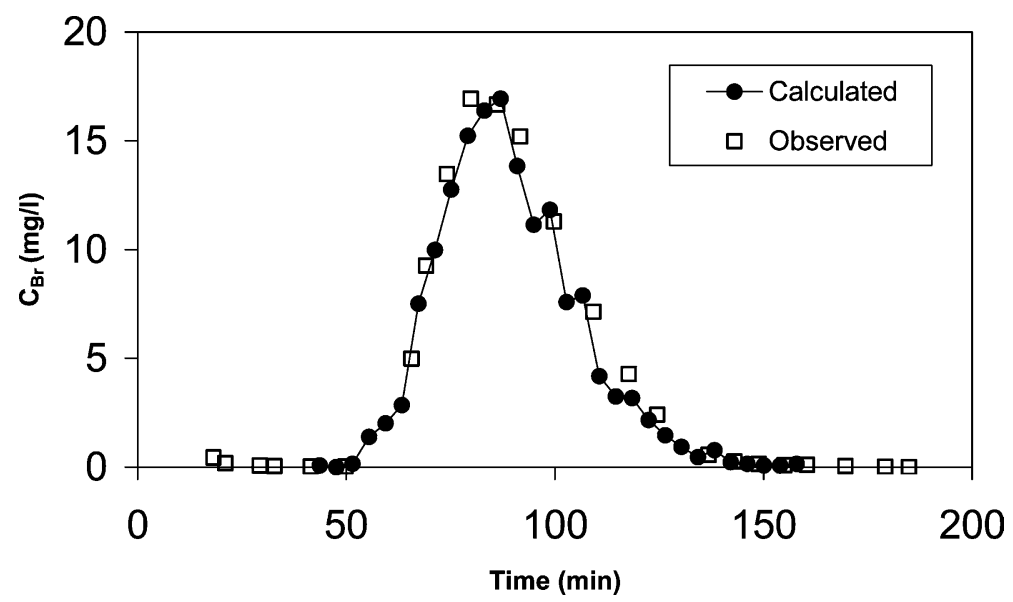

Fig. 5. Comparison of experimental and computational BTCs generated using the calibrated model. Breakthrough curve obtained from injection at port \#13. 
numerical model was adequately validated to conduct numerical simulations for further analysis.

\subsection{Results and analysis}

\subsubsection{Apparent porosity}

In analyzing the apparent porosity, a statistical univariate description was considered. $\phi_{\text {app }}$ values were interpreted as realizations of a single random variable without including the spatial location information (Table 3). It can be seen that even though the modelcalibrated effective porosity $\left(\phi_{\text {grid-scale }}=0.44\right)$ falls between the minimum and maximum $\phi_{\text {app }}$ values, it is overestimated by the geometric mean $\left(\sqrt{\phi_{\max } \phi_{\min }}\right)$. This discrepancy with the equivalent homogeneous theory can be explained by including the spatial information in the analysis. The $\phi_{\text {app }}$ color-scale map (Fig. 6a) illustrates how apparent porosities display a striking lack of elliptical behavior. Regions of low $\phi_{\text {app }}$ values seem to form irregular shapes strongly connected to the well. These regions of low $\phi_{\text {app }}$ values comprise transmissivities higher than the mean, and they define preferential channels through which particles may be favorably transported as they approach the well. The presence of these preferential channels makes the existence of any correlation between apparent porosity and directional transmissivity less apparent. A closer relation is expected from the equivalent continuum theory. These observations emphasize the fact that connectivity may mainly control the transport of solute in radially convergent flow. Connectivity is the intuitive concept that contemplates the presence in an aquifer of high conductivity pathways between the point of injection and the pumping well. Such pathways are thought to be dominated by point-transmissivity values that are greater than the average transmissivity of the total domain. In accordance with this reasoning, apparent porosity data from convergent-flow tracer tests seems to reveal zones of high connectivity to the well such that small apparent porosity values yield zones of high connectivity and vice versa.

The concept of connectivity was used by Sánchez-Vila et al. (1996) to explain the discrepancy in effective transmissivity between field data and stochastic theories based on multinormality of the log-conductivity fields. These authors suggest that the presence of

Table 3

Summary of univariate statistics

\begin{tabular}{lccr}
\hline & Ln $K(\mathrm{~m} /$ day $)$ & $\alpha_{\text {L,app }}(\mathrm{cm})$ & $\phi_{\text {app }}$ \\
\hline$N^{\text {a }}$ & 473 & 473 & 473 \\
Mean & 5.39 & 0.83 & 0.64 \\
0.25 -Quantile & 4.71 & 0.52 & 0.37 \\
Median & 5.92 & 0.69 & 0.61 \\
$0.75-Q u a n t i l e$ & 5.92 & 0.97 & 0.85 \\
MIN & 3.46 & 0.32 & 0.16 \\
MAX & 6.94 & 5.74 & 1.81 \\
Standard deviation & 1.12 & 0.50 & 0.31 \\
Coefficient of variation & 0.21 & 0.61 & 0.49 \\
Skewness & -0.21 & 3.84 & 0.48 \\
\hline
\end{tabular}

\footnotetext{
${ }^{a}$ Points located at the boundaries and at the well are not considered in the univariate statistical analysis.
} 

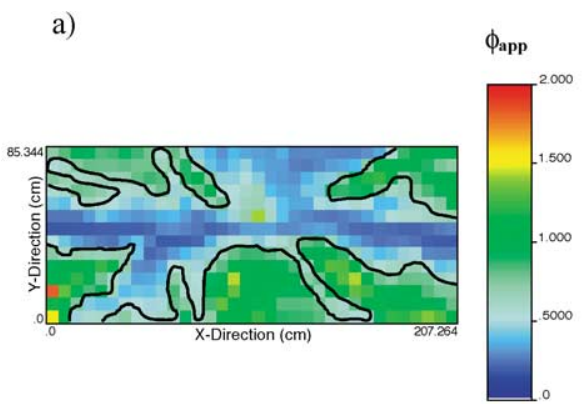

c)

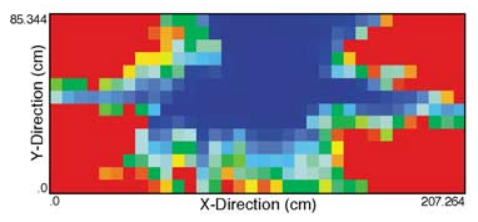

b)

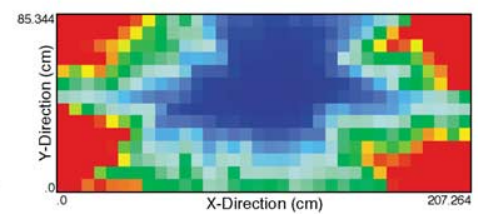

$\overline{\mathbf{t}}(\mathbf{m i n})$

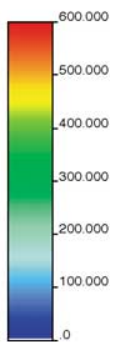

d)

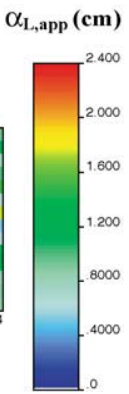

Fig. 6. Color-scale maps of (a) apparent porosity, preferential channels are highlighted by dark line, (b) arrival time, (c) second temporal moment, and (d) apparent longitudinal dispersivity.

regions with long connectivity would better explain most field results. Further numerical and analytical investigations conducted by Meier et al. (1998) and Sánchez-Vila et al. (1999), respectively, showed that the concept of connectivity better explains storativity estimates obtained from the interpretation of pumping test data assuming homogeneity (using the Theis or Jacob's method). Storativity from pumping tests is a function of the arrival time of the depression front at the observation well and may be seen as the analog parameter of apparent porosity. Therefore, both storativity estimates from pumping tests and porosity estimates from convergent-flow tracer tests seem to point to the fact that connectivity dominates solute transport under radially convergent flow.

It is important to recognize that based on these computational investigations, and by introducing the concept of connectivity, it is possible to explain some of the deviations found in the experimental data. It can be seen that the $\phi_{\text {app }}$ values of ports \#3 and \#20 are much higher than the neighbouring ports because they belong to regions of low connectivity. In contrast, ports \#24, \#15 and \#7 show much lower values because they are located within preferential channels even though they are close to ports \#3 and \#20.

\subsubsection{Analysis of temporal moments}

Temporal moments (Fig. $6 \mathrm{~b}$ and $\mathrm{c}$ ) show that the prior characteristics attributed to homogeneous fields are also manifested in the heterogeneous medium. The regions of equal 
$\sigma_{\mathrm{t}}^{2}$ and $\bar{t}$ values spatially delineate ellipses whose forms follow the main characteristics of the transmissivity field discussed in theoretical background. The results show that the temporal moments, in contrast to the $\phi_{\text {app }}$ behavior, are highly related to directional transmissivity and are not affected by the connectivity.

Apparent dispersivity (Fig. 6d) shows large spatial variations within the domain. This implies that caution must be taken when convergent-flow tracer techniques are used to estimate dispersivity. It is not clear what information of the aquifer is obtained from these dispersivity estimates. Nevertheless, it is worth to note that apparent dispersivity values appear to be higher in directions of high conductivity and vice versa. As discussed in Section 3, this was also observed in the tracer experiments. It should be pointed out that the intermediate-scale of experiments might be biasing some of the patterns and tendencies of apparent dispersivity, which may be better manifested at larger scales. The appearance of clearer tendencies on apparent dispersivity may be also obscured by boundary problems, which were suspected to be important at locations close to the well and to the constant head boundaries.

\section{Summary and conclusions}

Based on accurate experimental data generated in an intermediate-scale test tank and an experimentally validated numerical model, it was shown that in this particular test field, apparent porosity is more related to connectivity rather than to transmissivity. This puts into question the theoretical results obtained in equivalent fields in which effective porosity is assumed to be the geometric mean of the principal components of the apparent porosity tensor. It is concluded that in order to obtain meaningful effective porosity estimates from radial flow tracer tests, it is necessary to incorporate the underlying heterogeneous structure of the transmissivity field into the interpretation of the breakthrough curves.

It is also seen that the temporal moments follow similar rules as an equivalent homogeneous medium and are not influenced by the connectivity effect. Apparent dispersivity values are highly variable in the domain and spatial trends are not clear. Nonetheless, higher values are observed in directions of high conductivity and vice versa.

It should be noted that the conclusions that are derived in this work are based on tests conducted in an aquifer with the dimensions of the sand box. It is possible that connectivity became effective because of the dimensions. Still these types of analysis provide insights to the flow and transport behavior in naturally heterogeneous subsurface formations. Also, in view of the work by Chao et al. (2000), it is believed that the use of small sources in convergent-flow tracer tests may yield a sustained effect of connectivity at larger scales.

\section{Acknowledgements}

U.S. Army Research Office (Grant DAAD19-99-1-0195) and the Department of Geotechnical Engineering and Geosciences, Technical University of Catalonia provided the funding for this research. Equipment support was through Defense University Research 
Instrumentation Program (Grant DAAD19-99-1-0005). Jaime Gómez-Hernández and an anonymous reviewer provided very thorough and helpful comments and suggestions.

\section{References}

Barth, G.R., Illangasekare, T.H., Hill, M.C., Rajaram, H., 2001. Analysis of intermediate-scale tracer experiments for the development of tracer density guidelines. Water Resour. Res. 37 (1), 21-32.

Carrera, J., Walters, G.R., 1985. Theoretical developments regarding simulation and analysis of convergent-flow tracer tests: Technical Report for Sandia National Laboratories. Technical University of Catalonia, Barcelona, 572 pp.

Chao, 2000. Scale dependence of transport parameters estimated from forced-gradient tracer tests in heterogeneous formations. PhD Thesis, University of Colorado at Boulder, 208 pp.

Chao, C.-H., Rajaram, H., Illangasekare, T.H., 2000. Intermediate-scale experiments and numerical simulations of transport under radial flow in a two-dimensional heterogeneous porous medium. Water Resour. Res. 36 (10), 2869-2884.

Dagan, G., 1986. Statistical theory of groundwater flow and transport: pore to laboratory, laboratory to formation, and formation to regional scale. Water Resour. Res. 22 (9), 120S-134S.

Dagan, G., 1989. Flow and Transport in Porous Formations. Springer-Verlag, New York.

Delhomme, J.P., 1979. Spatial variability and uncertainty in groundwater flow parameters: a geostatistical approach. Water Resour. Res. 15 (2), 269-280.

Desbarats, A.J., 1992. Spatial averaging of transmissivity in heterogeneous fields with flow toward a well. Water Resour. Res. 28 (3), 757-767.

Fernàndez-Garcia, D., 1999. An experimental approach to convergent-flow tracer tests in heterogeneous media. Thesis dissertation, Technical University of Catalonia, $94 \mathrm{pp}$.

Freeze, R.A., 1975. A stochastic-conceptual analysis of one-dimensional groundwater flow in nonuniform homogeneous media. Water Resour. Res. 11 (5), 725-741.

Gelhar, L.W., 1986. Stochastic subsurface hydrology from theory to applications. Water Resour. Res. 22 (9), $135 \mathrm{~S}-145 \mathrm{~S}$.

Gelhar, L.W., Axness, C.L., 1983. Three-dimensional stochastic analysis of macrodispersion in aquifers. Water Resour. Res. 19 (1), 161-180.

Gelhar, L.W., Collins, M.A., 1971. General analysis of longitudinal dispersion in nonuniform flow. Water Resour. Res. 7 (6), $1511-1520$.

Gómez-Hernández, J.J., Gorelick, S.M., 1989. Effective groundwater model parameter values: influence of spatial variability of hydraulic conductivity, leakance and recharge. Water Resour. Res. 25 (3), 405-419.

Gómez-Hernández, J.J., Wen, X.-H., 1996. To be or not to be multi-Gaussian? A reflection on stochastic hydrogeology. Adv. Water Resour. 21 (1), 47-61.

Illangasekare, T.H., Armbruster, E.J., Yates, D.N., 1995. Non-aqueous-phase fluids in heterogeneous aquifersexperimental study. J. Environ. Eng. 121 (8), 571-579.

Kinzelbach, W., 1986. Groundwater Modeling: An Introduction with Sample Programs in BASIC. Elsevier, Amsterdam, The Netherlands, 333 pp.

Mackay, D.M., Freyberg, D.L., Roberts, P.V., Cherry, J.A., 1986. A natural gradient experiment on solute transport in a sand aquifer: 1. Approach and overview of plume movement. Water Resour. Res. 22 (13), $2017-$ 2029.

Meier, P.M., Carrera, J., Sánchez-Vila, X., 1998. An evaluation of Jacob's method for the interpretation of pumping tests in heterogeneous formations. Water Resour. Res. 34 (5), 1011-1025.

Moench, A.F., 1989. Convergent radial dispersion: a Laplace transform solution for aquifer tracer testing. Water Resour. Res. 25 (3), 439-447.

Sánchez-Vila, X., Carrera, J., 1997. Directional effects on convergent flow tracer tests. Math. Geol. 29 (4), $551-$ 569.

Sánchez-Vila, X., Colominas, I., Carrera, J., 1993. FAITH: User's Guide. Technical University of Catalonia, Barcelona, $50 \mathrm{pp}$.

Sánchez-Vila, X., Girardi, J.P., Carrera, J., 1996. Scale effects in transmissivity. J. Hydrogeol. 183, 1-22. 
Sánchez-Vila, X., Meier, P.M., Carrera, J., 1999. Pumping tests in heterogeneous aquifers: an analytical study of what can be obtained from their interpretation using Jacob's method. Water Resour. Res. 35 (4), $943-$ 952.

Sauty, J.P., 1980. An analysis of hydrodispersive transfer in aquifers. Water Resour. Res. 16 (1), 145-158.

Tompson, A.F.B., Gelhar, L.W., 1990. Numerical simulation of solute transport in three-dimensional, randomly heterogeneous porous media. Water Resour. Res. 26 (10), 2541-2562.

Wen, X.-H., 1995. Geostatistical methods for prediction of mass transport in groundwater. PhD Thesis, Kungl Tekniska Högskolan, Stockholm.

Wen, X.-H., Gómez-Hernández, J.J., 1996. The constant displacement scheme for tracking particles in heterogeneous aquifers. Ground Water 34 (1), 135-142. 